\section{La revisión de manuscritos en la Revista Médica de Chile y sus revisores externos en el año 2013}

\author{
HUMBERTO REYES B. ${ }^{1}$, MAX ANDRESEN H. ${ }^{2}$, JOAQUÍN PALMA H. ${ }^{2}$
}

\section{The manuscripts' review process in Revista Médica de Chile and its peer-reviewers during the year 2013}

\begin{abstract}
Mainstream journals, whether printed or electronic, are characterized among other standards- by the use of external peer review to critically assess those manuscripts that have been submitted for publication. The editors usually rely on peer reviewers' reports although their decisions are independent and usually final. The mission of Revista Médica de Chile includes the education of authors by guiding and stimulating them to improve their manuscripts before its acceptance and publication. Therefore the criticism, advice, and specific suggestions contained in peer reviewers' reports are a crucial tool to fulfill this mission. The present article indicates that during the year 2013, 422 persons participated as peer-reviewers of one or more manuscripts. Their names are listed in recognition of their voluntary contribution to the quality of this journal.
\end{abstract}

(Rev Med Chile 2014; 142: 909-913)

Key words: Journalism, medical; Peer review; Periodicals as topic.
${ }^{1}$ Editor.

${ }^{2}$ Editor Asociado.
Conflictos de intereses: Los autores son funcionarios de la Sociedad Médica de Santiago Sociedad Chilena de Medicina Interna, entidad propietaria de la Revista Médica de Chile que no tuvo influencia en la génesis del artículo, su redacción ni la decisión de publicarlo.
$\mathrm{E}$ 1 proceso de revisión de los manuscritos que se reciben en la Revista Médica de Chile sigue etapas secuenciales para su aceptación y publicación, o su rechazo. Estas etapas fueron resumidas en el Artículo Especial publicado en julio de 2013 (Rev Med Chile 2013; 141 (7): 903-908), pero nos parece útil reiterarlas y actualizarlas:

$1^{\text {a }}$ Recepción del manuscrito en la plataforma web, lo que permite a las secretarias verificar si cumple los requisitos formales solicitados en las Instrucciones a los Autores, incluyendo los documentos complementarios, tales como Guía de Exigencias para los Manuscritos, Declaración de Responsabilidad de Autoría, Declaración de Potenciales Conflictos de Intereses.

$2^{\text {a }}$ Revisión inicial del manuscrito por uno o más editores, quien(es) decide(n) si el tema abordado es pertinente para esta Revista y si el manuscrito incluye información adecuada y suficiente para continuar el proceso. En tal caso, las secretarias solicitan el pago por Recepción de Manuscritos-si corresponde-y la etapa siguiente espera a que este pago sea certificado por el autor corresponsal, mediante correo electrónico.

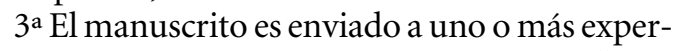
tos, identificados como revisores externos o "peer reviewers". El informe de los revisores externos es evaluado por los editores, quienes seleccionan una parte para darla a conocer a los autores, solicitándoles correcciones o modificaciones del manuscrito. A las observaciones de los revisores externos suelen agregarse otras del editor. Cuando los editores consideran que las críticas de los revisores son de tal importancia que no ameritan un intento de mejorar el manuscrito, lo rechazan en esta etapa del proceso.

A partir de julio de 2014 se advierte a los autores que tienen un plazo para reenviar una versión corregida. Si se recibe un manuscrito que ha so- 
brepasado este plazo, se le clasifica "nuevo" y por ende inicia todo el proceso desde su primera etapa.

$4^{\text {a }}$ Una vez recibida una versión corregida es reenviada a los revisores externos originales, para conocer su conformidad con el nuevo texto.

Los editores pueden obviar esta etapa si consideran que los cambios hechos al manuscrito y las explicaciones dadas por los autores son satisfactorios; en este caso se enviará una carta a los revisores explicando que se estimó innecesario solicitarles una segunda revisión.

$5^{\text {a }}$ Los editores deciden aceptar o no la publicación del manuscrito, lo que se comunica a los autores por correo electrónico.

6a Revisión final del manuscrito por uno de los editores, adecuando su formato al estilo de la Revista. En esta etapa pueden surgir críticas que hagan necesario solicitar nuevos cambios o aún motivar su rechazo, a pesar de haber sido previamente comunicada su aceptación. Esta última eventualidad es excepcional y siempre se informa a los autores y a los revisores la o las causas que motivaron esta decisión.

Las características principales de este proceso son similares para las revistas médicas que emplean la revisión por pares y el rol de los revisores externos es importante. De ellos se espera que respondan prontamente si aceptan o no hacer la revisión y que declaren si tienen conflictos de intereses con el manuscrito o con sus autores. $\mathrm{Si}$ aceptan hacer la revisión, se les solicita que entreguen su informe en un plazo breve (dos semanas), salvo en situaciones especiales que deberán autorizar los editores. Sus comentarios, particularmente aquellos que serán transmitidos a los autores, deberían ser constructivos, honestos y corteses. En esta Revista se respeta el anonimato de los revisores frente a los autores.

La "revisión por pares" es uno de los pilares que sostienen la calidad de las revistas científicas y se ha hecho cada día más difícil al aumentar la complejidad metodológica de los trabajos, tanto en el área científica como en las especialidades clínicas. Esta colaboración voluntaria y desinteresada demanda tiempo, esfuerzo, la revisión de bases de datos y de otras publicaciones, y la aplicación de un juicio valorativo, todo lo cual se sintetiza en un informe. Es un trabajo que debe ser apreciado por los lectores y por las instituciones en que trabajan dichos revisores. Por ello, una vez al año y en el número de julio de la Revista, se publican los nombres de las personas que revisaron manuscritos durante el año calendario precedente. Durante 2013 contamos con la participación de 422 revisores externos, la mayoría de los cuales revisaron dos o más manuscritos.

En nombre de los editores de la Revista Médica de Chile y del Directorio de la Sociedad Médica de Santiago-Sociedad Chilena de Medicina Interna, agradecemos a quienes revisaron manuscritos durante el año 2013. La nómina que se publica a continuación es un documento que acredita su participación en el proceso de "revisión por pares".

\section{Listado revisores 2013}

\author{
Katia Abarca V \\ Fernando Abarzúa C \\ Patricia Abumohor $\mathrm{G}$ \\ Mónica Acevedo B \\ Cristián Acevedo C \\ Raúl Acuña $M$ \\ Mónica Acuña P \\ Rubén Aguayo N \\ María Luisa Aguirre C \\ Mónica Ahumada O \\ Francisco Alarcón A \\ Ana María Alarcón M \\ Sylvia Alegría Q
}

Jorge Alfaro L

Angélica Alliende R

María Alvarado B

Manuel Álvarez L

Manuel Álvarez Z

Miriam Alvo A

Roberto Amador C

Cristián Amador C

Hugo Amigo C

Werner Apt B

Francisco Arancibia $\mathrm{H}$

Juan Pablo Arancibia P

Teresa Aravena C

\author{
Pablo Aravena C \\ Carlos Aravena L \\ Fernando Araya D \\ Héctor Araya I \\ Iván Araya $\mathrm{H}$ \\ Leopoldo Ardiles A \\ Iván Armijo R \\ Pilar Arnaiz G \\ Oscar Arteaga $\mathrm{H}$ \\ Antonio Arteaga Ll \\ Eugenio Arteaga U \\ Carmen Astete A \\ Eduardo Atalah S
}


Arturo Ayala B

Carmen Gloria Aylwin H

Marcela Babul K

Lautaro Badilla O

Ramón Baeza B

Cristián Baeza $\mathrm{P}$

Ricardo Baeza V

Claudia Bambs S

Salesa Barja Y

Alejandro Barrera E

René Mauricio Barria $\mathrm{P}$

Manuel Barros M

María de la Luz Bascuñán $\mathrm{R}$

Gabriel Bastías S

Luis Bavestrello F

Paula Bedregal G

Ernesto Behnke G

Pablo Bernier B

Pablo Bertín C-M

Carmen Gloria Bezanilla C

Leandro Biagini A

Víctor Bianchi Sch

Alex Bittner B

Yerko Borghero R

Pablo Brockmann V

Lucía Bronfman F

Daniel Bunout B

Paula Burgos C

Francisco Bustamante V

Jorge Calderón P

Jamile Camacho N

Jorge Carabantes C

Gloria Calaf M

César Cárcamo Q

Carlos Caorsi S

María Capetillo F

Daniel Capurro N

Leonardo Carmona $\mathrm{R}$

Carmen Carrasco M

Marcela Carrasco G

Fernando Carrasco N

Víctor Carrasco M

Yuri Carvajal B

Carlos Carvajal H

Aurelio Carvallo V

José Luis Castillo C

Manuel Castillo N

Octavio Castillo C
Carla Castillo L

Silvia Castillo T

Pablo Castro G

Juan Céspedes G

Hernán Chamorro B

Gastón Chamorro S

Lucía Cifuentes O

Miguel Ángel Clerc C

Beatriz Comparini F

Héctor Contreras M

Jaime Contreras $\mathrm{P}$

Luis Contreras M

Andrea Contreras St

Ramón Corbalán H

María Patricia Cordella M

Gerardo Correa I

Pelayo Correa V

Julio Cortés C

Fanny Cortés M

Víctor Cortés M

Alejandro Corvalán R

Eduardo Cosoi P

José Manuel Cousiño L

Carolina Cruz B

Adolfo Cruz C

Miguel Cuchacovich T

Francisco Cubillos R

Eduardo Cunill C

Ada Cuevas M

Jorge Dagnino S

Xabier De Aretxabala U

Flora de la Barra Mac D

María Pía De la Maza C

César Del Castillo S

Marta del Río A

Sergio Del Villar P-M

Carolina Delgado D

Fernando Descalzi M

Luigi Devoto C

Jaime Díaz C

Orlando Díaz P

Julio Dölz V

Otto Dörr Z

Patricio Downey C

Pilar Durruty A

Guillermo Durruty V

Gastón Dussaillant M

Carlos Echeverría B
Rodrigo Erazo R

Edgardo Escobar C

Manuel Espinoza S

Luis Espinoza $\mathrm{R}$

Ricardo Espinoza G

Alejandro Fajuri N

Patricia Fardella B

Rossana Faúndez H

Mauricio Fernández R

Gustavo Figueroa C

Ramón Florenzano U

Juan Carlos Flores $\mathrm{H}$

Claudio Flores $\mathrm{P}$

Alejandra Fuentes G

Homero Gac E

Gonzalo Gajardo G

Eugenio Galilea M

Pilar Gambra A

Bernardita Garayar P

Flavia Garbin A

María José García R

Juan Pablo García-H T

María Luisa Garmendia M

Gabino Garrido G

Héctor Gatica R

Ricardo Gazitúa $\mathrm{H}$

Alfredo Germain A

Rodrigo Gil D

Iván Godoy J

Gonzalo Godoy J

Alejandro Goic G

Alejandro Gómez Ch

Sergio González B

Fernando González F

Mónica González Y

Ignacio Goñi E

Martín Gotteland

Marcos Goycoolea V

Carlos Grant del R

Douglas Greig U

Juan Francisco Guerra C

Ernesto Guiraldes C

Mónica Gutiérrez N

María Antonieta Guzmán M

Jorge Hasbún $\mathrm{H}$

Ariel Hasson N

Andrés Heerlein L

Carlos Hermansen T 
Viviana Herskovic M

Pedro Herskovic L

Sandra Hirsch B

Lorena Hoffmeister A

Alvaro Huete G

Ricardo Hughes $G$

Bessie Hunter M

Humberto Ibarra $\mathrm{V}$

Juan Idiáquez $\mathrm{C}$

Hernán Iturriaga $\mathrm{R}$

Liliana Jadue $\mathrm{H}$

Jorge Jalil M

Roberto Jalil M

Alejandra Jara $\mathrm{G}$

Cristián Jensen $B$

Jorge Jiménez De la J

Jorge Jorquera A

Carlos Juri C

Gustavo Kaltwasser G

Christian Karmelic S

Ricardo Katz B

Alejandra King D

Julieta Klaassen L

Owen Korn B

Sonia Kunstmann F

Luigi Lagazzi G

Nestor Lagos F

Alexis Lama T

Martín Larico G

Marcela Larraguibel Q

Martín Francisco Lasso B

Jorge Lastra $\mathrm{T}$

Thomas Leisewitz V

Rony Lenz A

Francisco León C

Luz María Letelier S

Claudio Liberman G

Ximena Lioi C

Marcelo Llancaqueo V

Osvaldo Llanos L

Sergio Loayza $S$

Nelson Lobos J-F

Ilse López B

Francisco López K

José Manuel López M

Gloria López St

Jaime Lubascher C

Paul McNab M
Jorge Madrid A

Pedro Maggiolo G

Alberto Maíz G

Alejandro Majlis L

Eugenio Marchant D

Marcelo Marconi T

Alejandra Marcotti S

Paula Margozzini M

Guillermo Marshall R

Alejandro Martínez S

Jorge Martínez C

Virginia Martínez C

Benjamín Martínez R

María Eugenia Martínez R-E

Víctor Martínez Z

Pablo Matamala B

Percy Mayta-Tristán

Claudio Mella S

Cecilia Mellado S

Manuel Meneses C

Tomás Merino L

Renato Mertens M

Sergio Mezzano A

Patricio Michaud Ch

Pedro Miranda C

Marcelo Miranda C

Héctor Miranda V

Juan Molina Y

Ramiro Molina C

Rodrigo Montagna M

Gustavo Montaldo L

Martín Montecino L

Hernán Montenegro A

José Miguel Montes S

Helia Morales M

Raimundo Morris Cárdenas

Claudio Mosso Ch

Lorena Mosso G

Leonor Moyano Sch

Bettina Müller Sch

Patricia Muñoz C del V

Gabriela Muñóz G

Sergio Muñoz N

Fernando Muñoz P

Silvana Muñoz S

Evelyn Muñoz S

Santiago Muzzo B

Oscar Neira Q
Luis Miguel Noriega $\mathrm{R}$

Victoria Novik A

Fernando Novoa S

Mauricio Ocqueteau T

Danny Oksenberg R

Verónica Olavarría L

Alfonso Olmos C

Luis Orlandi J

Rodrigo Orozco B

Ana Mireya Ortíz M

Manuel Ortíz P

Miguel O'Ryan G

Manuel Oyarzún G

Alejandra Palma B

Cristián Palma C

María Teresa Parada C

Mauro Parra C

Luis Pedraza C

Jacqueline Pefaur $\mathrm{P}$

Blanca Peñaloza H

María Luisa Pérez-Cotapos S

Carlos Pérez C

Julio Pertuzé R

Pedro Pineda B

Paulina Pino Z

Ignacio Pinto Q

Tito Pizarro Q

Felipe Pollak C

Jaime Poniachik T

Juan Carlos Prieto D

Ivan Quevedo L

Juan Carlos Quintana F

Mónica Quintana C

Francisco Radrigán A

Danuta Rajs G

Jorge Rakela B

Pablo Ramírez V

Andrés Reccius M

Carlos Regonesi M

Paula Repetto L

Pedro Retamal C

Ricardo Rey C

Alvaro Reyes $\mathrm{P}$

José Miguel Reyes V

Enrique Reynolds $\mathrm{H}$

José Ribalta $S$

Ximena Riesco C

Attilio Rigotti R 
Arnoldo Riquelme P Juan Carlos Roa St José Ignacio Rodríguez C Juan Carlos Rodríguez D Emilio Roessler B Christine Rojas $\mathrm{H}$ Antonio Rollán R Oscar Román A Rossana Román R Carlos Romero P Paula Rothhammer A Jaime Rozowski N Elsa Rugiero P Mauricio Ruiz C Sofía Salas P Claudio Salas F Sergio Salas F Jessica Salinas L Rodrigo Salinas R Karen Salvo C Hugo Sánchez R Patricio Sandoval R Edgar Sanhueza B Patricio Sanhueza C Gabriel Sanhueza C Emilia Sanhueza R Jaime Andrés Santander T Manuel Santos A Ricardo Schwartz J Daniel Seijas B Roberto Segovia M Rafael Selman A
Rafael Sepúlveda J

Alejandro Serani M

Héctor Serrat K

Beatriz Shand K

Francisco Silva L

Juan Jorge Silva S

Patricio Smith F

Raúl Smith P

Viviana Sobrero R

Iván Solis O

Marco Antonio Soza M

Benjamín Stockins F

Mauricio Tagle M

Alvaro Téllez T

Eduardo Tobar A

Vinko Tomicic F

Carlos Toro A

Marisa Torres H

Patricio Torres A

Roberto Torres U

Ximena Triviño B

Cristian Trujillo L

Sergio Trujillo V

Eduardo Turner G

Diana Ulloa M

Mario Uribe $\mathrm{M}$

Julio Urrutia E

Jose Rafael Valbuena M

Gloria Valdés St

Gonzalo Valdivia C

Vicente Valdivieso D

Andrés Valdivieso D
Sergio Valdivieso F

María Teresa Valenzuela B

Jorge Valenzuela E

Regina Vargas R

Luis Vega B

Alfredo Velasco P

Pablo Vera V

Juan Verdaguer $\mathrm{T}$

María Teresa Vergara A

Marcos Vergara I

Carmen Gloria Vergara L

Consuelo Vergara R

Fernando Vergara E

Wilson Vielma A

Pablo Vigorena $\mathrm{S}$

Leonor Villacura A

Luis Villarroel del P

Claudio Villarroel Q

Paulina Villaseca D

Eduardo Wainstein G

Ronald Wainstein G

Kristina Weil P

Nelson Wohllk G

Masami Yamamoto C

Ricardo Zalaquett S

Jaime Zamorano $G$

Beatriz Zegers $\mathrm{P}$

Pedro Zitko M

Carlos Zúñiga $S$

Denisse Zúñiga $P$

György Szánthó P 